\title{
Semi-blind Iterative Joint Channel Estimation and K-Best Sphere Decoding for MIMO
}

\author{
I. Dey, G. G. Messier and S. Magierowski \\ School of Electrical and Computer Engineering \\ University of Calgary \\ Calgary, Canada \\ Email: deyi@ucalgary.ca, gmessier@ucalgary.ca, smagiero@ucalgary.ca
}

\author{
Sheng Chen \\ Electronics and Computer Science \\ Faculty of Physical and Applied Sciences \\ University of Southampton \\ Southampton, UK \\ Email:sqc@ecs.soton.ac.uk
}

\begin{abstract}
An efficient and high-performance semi-blind scheme is proposed for Multiple-Input Multiple-Output (MIMO) systems by iteratively combining channel estimation with K-Best Sphere Decoding (SD). To avoid the exponentially increasing complexity of Maximum Likelihood Detection (MLD) while achieving a near optimal MLD performance, K-best SD is considered to accomplish data detection. Semi-blind iterative estimation is adopted for identifying the MIMO channel matrix. Specifically, a training-based least squares channel estimate is initially provided to the K-best SD data detector, and the channel estimator and the data detector then iteratively exchange information to perform the decision-directed channel update and consequently to enhance the detection performance. The proposed scheme is capable of approaching the ideal detection performance obtained with the perfect MIMO channel state information.
\end{abstract}

Index Terms-Multiple-input multiple-output, K-Best Sphere Decoding, joint channel estimation and data detection

\section{INTRODUCTION}

Data detection techniques for Multiple-Input MultipleOutput (MIMO) systems proposed over the last few years include linear Zero Forcing (ZF) and Minimum Mean Square Error (MMSE) detectors as well as nonlinear Maximum Likelihood (ML) detection [1], [2]. Of these, the ML decoder (MLD) offers the optimal performance at the expense of exponential computational complexity. In order to approximate the optimal performance, but at a much lower computational complexity, a set of nonlinear data detectors called Sphere Decoders (SDs) were introduced in [3], [4]. SDs can not only support hard-decision data detection, but also can be extended to soft-decision decoding to support channel coded MIMO system [5]. An improvement over the traditional SD was presented in [6], where a technique based on the breadthfirst search algorithm is applied, K-Best SD (KSD). It offers the advantage of allowing only $K$ best candidates to proceed to the next level, thereby reducing computational complexity. With an appropriately chosen value for $K, \mathrm{KSD}$ is capable of approaching the optimal ML detection performance at a much lower complexity. KSD can also be extended to support soft outputs, as in List SD (LSD) of [7] and List Sequential SD (LISS) of [8]. A posteriori probability (APP) detection follows sphere decoding to detect data on the receiver side.

The ability of a coherent MIMO system to approach Shannon's capacity bound highly depends on the accuracy of the channel state information (CSI). Adaptive MIMO systems perform optimization on the basis of the chosen channel estimator used to provide the CSI for data detection. Several channel estimation algorithms have been proposed for adaptive MIMO systems, which can be broadly categorized as trainingbased, blind and semi-blind algorithms. Training-based adaptive schemes can accurately estimate a MIMO channel, but at the cost of large training overhead which reduces the achievable system throughput. Blind methods exhibit high complexity, slow convergence and languish from unavoidable estimation and decision ambiguities [9]. Semi-blind methods implement decision-directed channel estimation, where a few training symbols are used to provide an initial MIMO channel estimate [10] to help to resolve the estimation and decision ambiguities suffered by pure blind schemes.

The semi-blind approach typically employs joint channel estimation and data detection through iterative exchange of information between the channel estimator and the data detector. Joint semi-blind channel estimation and ML data detection has long been a fixture in the literature. A large number of iterations is required by the channel estimator. High-complexity MLD amplifies the computational complexity of such an algorithm to a prohibitively high level. MLD has been replaced with a simpler SD for Space-Time Block Codes (STBC) in [11] and rank-deficient MIMO systems in [12]. However, these SDs suffer from a non-fixed decoding complexity over varying SNR values and the channel estimation requires a large number of iterations to achieve convergence.

This paper proposes a low-complexity joint semi-blind iterative channel estimation and KSD data detection algorithm for MIMO systems. A minimal number of training blocks, which is related to the number of transmit antennas, are first used to provide an approximate Least Square Channel Estimate (LSCE). Based on this initial LSCE, data is detected using KSD. The detected data is then remodulated and used for the decision-directed LSCE update. The performance and complexity of the proposed joint channel estimator and data detector is investigated using simulation, and the results obtained show that a very few iterations, around four or five, are sufficient for the proposed semi-blind scheme to approach optimal detection performance with perfect CSI.

The remainder of this paper is organized as follows. Sec- 
tion II describes the MIMO system model and our proposed joint semi-blind iterative channel estimation and KSD algorithm for hard-decision decoding, and the simulation results are presented in Section III, while our conclusions are offered in Section IV.

\section{System Model And Proposed Scheme}

\section{A. MIMO Model and K-Best Sphere Decoder}

Consider the MIMO system consisting of $M_{t}$ transmit antennas and $M_{r}$ receive antennas. The communication channel is assumed to be narrowband or flat Rayleigh faded and quasistationary. The overall MIMO system can be modeled using the equation, $\mathbf{Y}(k)=\mathbf{H} \mathbf{S}(k)+\mathbf{N}(k)$, where, $k$ denotes the symbol index, $\mathbf{H} \in \mathbb{C}^{M_{r} \times M_{t}}$ is the MIMO channel matrix, $\mathbf{Y} \in \mathbb{C}^{M_{r}}$ and $\mathbf{S} \in \mathbb{C}^{M_{t}}$ represent the received and transmitted signal vectors, respectively, while $\mathbf{N}$ is the complexvalued Additive White Gaussian Noise (AWGN) vector with $\mathrm{E}\left[\mathbf{N N}^{\mathrm{H}}\right]=2 \sigma_{N}^{2} \mathbf{I}_{M_{r}}$ and $\mathbf{I}_{M_{r}}$ being an $M_{r} \times M_{r}$ identity matrix.

Based on the above system model, a lower complexity lattice decoder like K-Best SD can be implemented for data detection at the receiver. It operates on breadth-first search [13], [14]. A hyper-sphere of radius $\sqrt{C}$ is chosen around the received signal vector. The search algorithm will traverse through all the points within this sphere. At each layer of the search tree, only a fixed $K$ Partial Euclidean Distances (PEDs) survive. The sorting process continues with $K$ smaller PEDs retained from the previous layer. Progressing in this way, the node with the smallest accumulated PED at the last layer of the search tree will be considered as the optimal ML solution.

\section{B. Semi-blind Iterative Channel Estimation and K-Best SD}

Our main contribution is to develop a joint semi-blind iterative channel estimator and KSD. Assume that initially $M$ training blocks are available. The received training data is recorded as, $\mathbf{Y}_{M}=[\mathbf{y}(1) \mathbf{y}(2) \cdots \mathbf{y}(M)]$ and the transmitted training information as, $\mathbf{S}_{M}=[\mathbf{s}(1) \mathbf{s}(2) \cdots \mathbf{s}(M)]$. Then the LSCE of the channel matrix $\mathbf{H}$ based on the training data $\mathbf{Y}_{M}$ and $\mathbf{S}_{M}$ is given by,

$$
\check{\mathbf{H}}_{\mathrm{LSCE}}=\mathbf{Y}_{M} \mathbf{S}_{M}^{\mathrm{H}}\left(\mathbf{S}_{M} \mathbf{S}_{M}^{\mathrm{H}}\right)^{-1}
$$

To maintain the system throughput, a minimum number of training blocks $M$ should be used. In order for $\mathbf{S}_{M} \mathbf{S}_{M}^{\mathrm{H}}$ to have a full rank, it is necessary that $M \geq M_{t}$, and it follows that a minimum number of training blocks is $M=M_{t}$. For example, if $M_{t}=4$, the minimum number of training blocks required is $M=4$.

Semi-blind Iterative Algorithm: Assume that each symbol packet consists of $\tau$ information symbols. Then the observation matrix for the KSD to detect is represented as, $\mathbf{Y}_{\tau}=$ $[\mathbf{y}(1) \mathbf{y}(2) \cdots \mathbf{y}(\tau)]$. Given the fixed number of iterations, $I_{\max }$, the proposed semi-blind iterative algorithm can now be summarized.

STEP 1: The iteration index is initialized to $i=0$ and the initial channel estimate is set to $\widehat{\mathbf{H}}^{(i)}=\check{\mathbf{H}}_{\mathrm{LSCE}}$.
STEP 2: Given $\widehat{\mathbf{H}}^{(i)}$, the KSD performs the data detection on $\mathbf{Y}_{\tau}$ to yield $\widehat{\mathbf{S}}_{\tau}^{(i)}=\left[\widehat{\mathbf{S}}^{(i)}(1) \widehat{\mathbf{s}}^{(i)}(2) \cdots \widehat{\mathbf{S}}^{(i)}(\tau)\right]$, where $\widehat{\mathbf{s}}^{(i)}(k)$ denotes the remodulated detected symbol vector at the $i$ th iteration for the transmitted symbol vector $\mathbf{s}(k)$.

STEP 3: The channel estimate is then updated with,

$$
\widehat{\mathbf{H}}^{(i+1)}=\mathbf{Y}_{\tau}\left(\widehat{\mathbf{S}}_{\tau}^{(i)}\right)^{\mathrm{H}}\left(\widehat{\mathbf{S}}_{\tau}^{(i)}\left(\widehat{\mathbf{S}}_{\tau}^{(i)}\right)^{\mathrm{H}}\right)^{-1}
$$

STEP 4: Set $i=i+1$. If $i<I_{\max }$, the algorithm goes back to STEP 2; Otherwise it is stopped.

The total complexity of this procedure is proportional to the product of the maximum number of iterations, $I_{\max }$ and the decoding complexity of the KSD. If the modulation constellation size is $L$, it can be shown that an appropriate choice for $K$ is $K=L$. As a result, the KSD has to search for a total of $\tau \times\left(L+\left(M_{R}-1\right) L^{2}\right)$ constellation points. Cholesky factorization also imposes some extra complexity.

\section{Simulation Results}

The performance of the proposed joint semi-blind iterative channel estimation and K-best SD algorithm is evaluated using a $M_{t}=M_{r}=4$ MIMO system with the Quadrature Phase Shift Keying (QPSK) modulation scheme. All the simulation results are averaged over 100 independent random channel realizations of the flat Rayleigh channel environment. Since $L=4$ for the QPSK modulation scheme, we choose $K=4$ and $C$ is empirically chosen as, $C=10$. The Signal-to-Noise Ratio (SNR) of the simulation system is defined as SNR = $\sigma_{S}^{2} / 2 \sigma_{N}^{2}$, where $\sigma_{S}^{2}$ is the average symbol energy. The data frame length $\tau$ is chosen to be $\tau=100$.

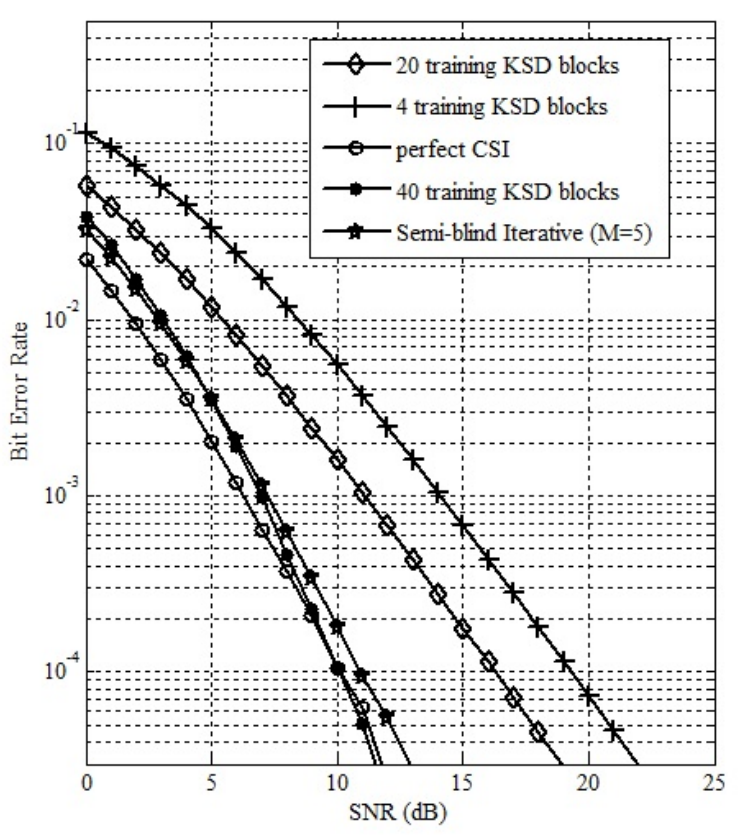

Fig. 1. Bit error rate of the proposed semi-blind scheme with $M=5$ initial training symbols, in comparison with the training-based cases using 4, 20 and 40 training symbols, respectively, as well as the case of perfect channel state information. 


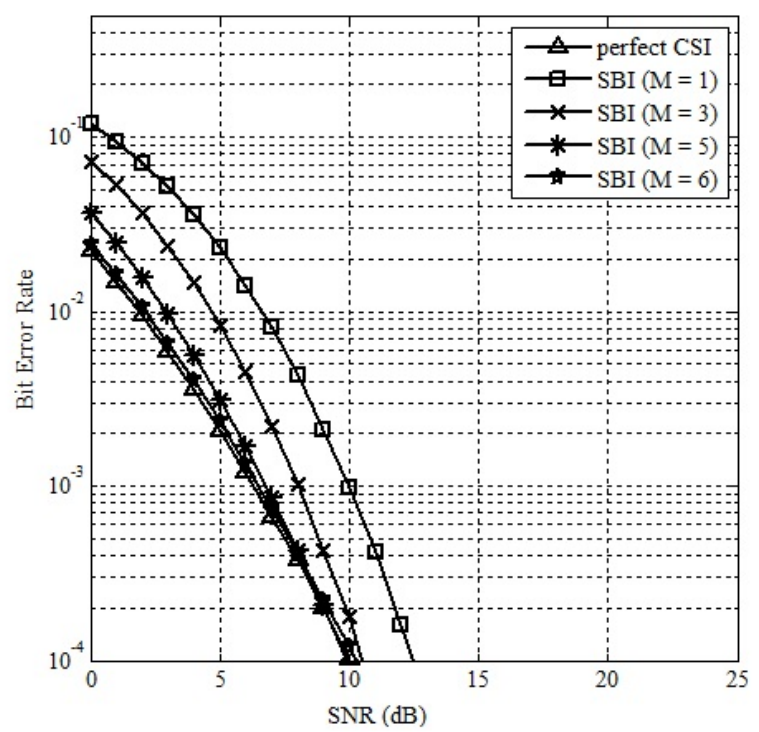

Fig. 2. Comparative bit error rate performance of the proposed semi-blind scheme with $M=1,3,5$ and 6 initial training symbols, respectively, along with the case of perfect channel state information.

The BER performance of KSD with the perfect CSI is depicted in Fig. 1 as the benchmark of the achievable performance, where the BERs of KSD based on the channel estimates obtained with 4,20 and 40 training symbols, respectively, are also plotted for comparison. It can be seen from Fig. 1 that a training length of $M=4$ offers performance far below K-best sphere decoding with perfect CSI. Performance equivalent to perfect CSI is only achieved with $M=40$. In contrast, our proposed semi-blind iterative (SBI) algorithm can meet or exceed the BER performance of conventional KSD using LSCE and $M=40$. This is achieved with only $M=5$

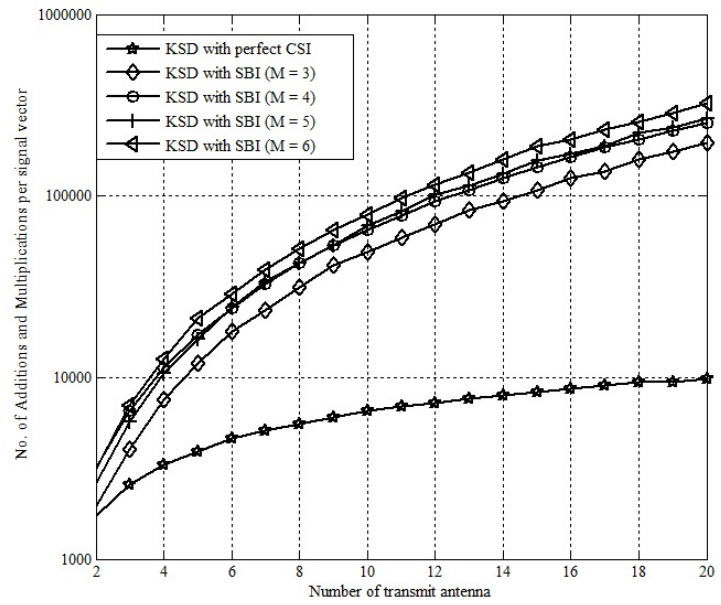

Fig. 3. Computation complexity of the proposed semi-blind scheme with $M=3,4,5$ and 6 initial training symbols, respectively, in comparison with the case of perfect channel state information, plotted over the number of transmit antennas.

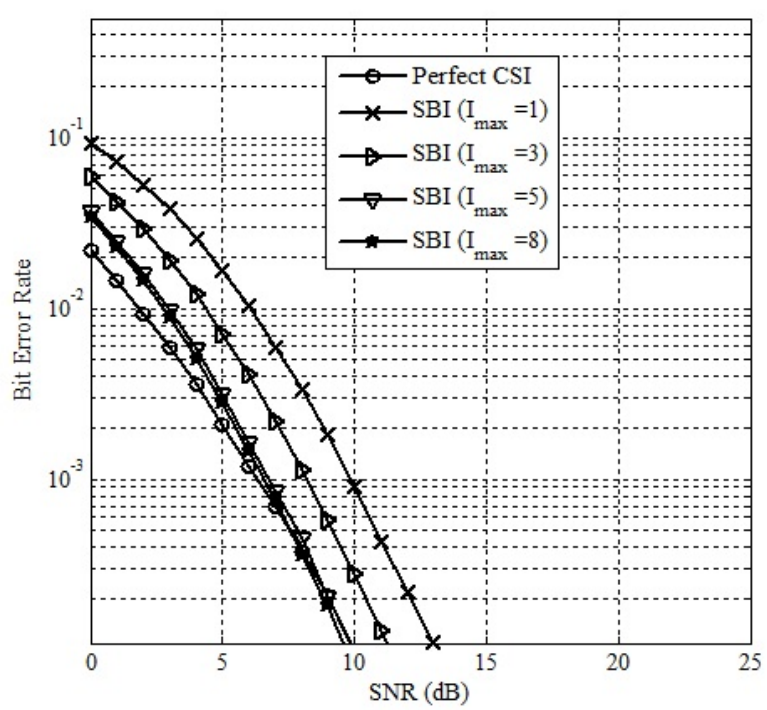

Fig. 4. Comparative bit error rate performance of the proposed semi-blind scheme with maximum number of iterations bound to, $I_{\max }=1,3,5$ and 8 , respectively, along with the case of perfect channel state information.

initial training blocks and $I_{\max }$ set to 4 . This demonstrates that the proposed iterative scheme improves the channel estimate with each iteration, which in turn improves the detection performance of KSD.

It can be seen from Fig. 1 that the semi-blind iterative algorithm with only $M=5$ initial training blocks approaches

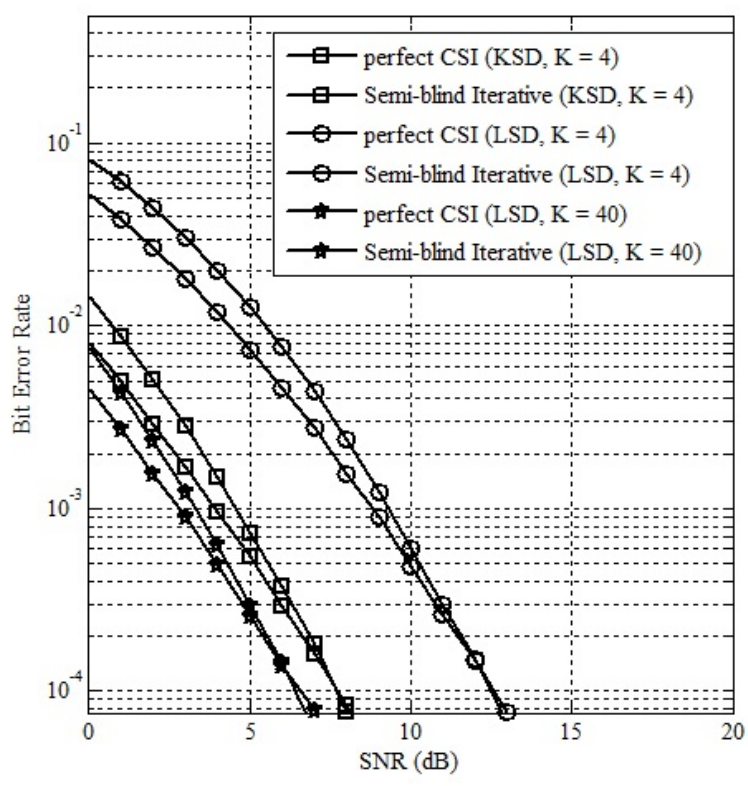

Fig. 5. Comparative bit error rate performance of the proposed semi-blind scheme with $M=5$ initial training symbols, employed to $1 / 2$-rate outer Convolutional coded, K-best SD $(K=4)$ with hard-decision decoding and List SD ( $K=4$ and $K=40$ ) with soft-decision decoding respectively, as well as the cases of perfect channel state information. 
the optimal detection performance of KSD with perfect CSI for $\mathrm{SNR} \geq 9 \mathrm{~dB}$. By increasing the initial training blocks to $M=6$, better performance can be achieved. Fig. 2 illustrates the effect of increasing $M$ on the performance of the semiblind iterative algorithm. The figure shows that $M=6$ initial training blocks achieves the optimal performance of KSD with perfect CSI for SNR $\geq 7 \mathrm{~dB}$. Of course, increasing $M$ also increases the complexity of the algorithm, as illustrated in Fig. 3

Fig. 3 depicts the computation complexity of the KSD with perfect CSI, in comparison with the proposed joint semiblind iterative channel estimation and KSD schemes over the number of transmit antennas. The initial number of training blocks is gradually increased from $M=3$ to $M=4,5$ and 6 respectively. A constant SNR of $10 \mathrm{~dB}, K=4$ and $I_{\max }=4$ are maintained. Note that for a $4 \times 4$ MIMO system, there are $M_{t} \cdot M_{r}=16$ complex-valued channel taps, or 32 real-valued channel coefficients. $M=4$ training blocks correspond to 32 training bits and, therefore, only a training overhead of 1 bit per channel coefficient was used for the case of $M=4$. If $M=5$ initial training blocks were chosen, the training overhead remained approximately 1 bit per channel coefficient and the complexity remains essentially unchanged. However, as $M$ increases to 6 , the training overhead approximately increases to 2 bits per channel coefficient, thereby increasing the computational complexity, as can be seen in Fig. 3 .

Increasing the maximum number of iterations allowed, results in the improvement of performance as can be observed in Fig. 4. A constant $M=5$ initial training blocks is used in generating the results in Fig. 4. A large improvement in performance is observable when $I_{\max }$ is increased from 1 to 3 , while a minor enhancement is revealed with $I_{\max }$ increasing from 5 to 8 . Hence, increasing the maximum number of iteration achieves significant performance improvement, only if $I_{\max } \leq 5$.

Fig. 5 depicts the BER performances of the proposed semiblind scheme with $M=5$ initial training symbols, when used with a $1 / 2$-rate outer convolutional code. K-Best sphere decoding performance with $K=4$, and hard-decision Viterbi decoding has been compared with list sphere decoding with $K=4$ and $K=40$, and APP soft-decision decoding. In each case, the performance of the proposed channel estimation algorithm has been plotted along with that of the perfect CSI benchmark. Comparing the results in Fig. 1 and Fig. 5, we can see that application of channel coding improves the performance of KSD with $K=4$ by around $3 \mathrm{~dB}$. But LSD with $K=4$ yields a loss in performance by around $6 \mathrm{~dB}$ as compared with $\mathrm{KSD}$ with $K=4$. LSD performance can surpass KSD but only with considerable additional complexity, as shown by the $K=40$ results in Fig. 5. Therefore, softdecision decoding with LSD enhances performance over KSD with hard-decision decoding and outer channel coding, but at the expense of larger computational complexity. However, in each case (i. e. KSD with $K=4$, LSD with $K=4$ and LSD with $K=40$ ), our proposed semi-blind iterative algorithm approaches the benchmark with small number of iterations and minimum training overhead.

\section{CONCLUSION}

The major challenge in the implementation of MIMO systems results from inter-antenna interference in spatial multiplexing and imperfect CSI affecting the MIMO detection of information bits at the receiver side. The K-best sphere decoder is known to approach optimal ML detection at a much lower complexity, given perfect CSI. This paper has proposed a joint semi-blind channel estimator and K-best sphere detection scheme for MIMO systems, which requires a minimum training overhead and yet is capable of approaching the optimal detection performance obtained with perfect CSI. Specifically, the channel estimator and the data detector recursively exchange information to enhance channel estimation accuracy and attain optimal detection performance with a very small number of iterations. Simulation results also confirm that our proposed approach achieves this performance with very low complexity and training overhead.

\section{REFERENCES}

[1] D. Garrett, L. Davis, S. T. Brink, and B. Hochwald, "APP processing for high performance MIMO systems," in Proc. IEEE Custom Integrated Circuits Conference, San Jose, CA, Sep. 2003, pp. 271-274.

[2] A. Burg, N. Felber, and W. Fichtner, "A 50 Mbps 4X4 maximum likelihood decoder for multiple-input multiple-output systems with QPSK modulation," in Proc. 10th IEEE Int. Conf. Electron., Circuits, Syst. (ICECS), Dec. 2003, pp. 332-335.

[3] M. O. Damen, K. Abed-Meraim, and J. C. Belfiore, "A generalized lattice decoder for asymmetrical space-time communication architecture," in Proc. IEEE Acoustics, Speech, and Signal Processing (ICASSP), Jun. 2000, pp. 2581-2584.

[4] B. Hassibi and H. Vikalo, "On the expected complexity of sphere decoding," in Thirty-Fifth Asilomar Conference on Signals, Systems and Computers, Nov. 2001, pp. 1051-1055.

[5] B. M. Hochwald and S. T. Brink, "Achieving near-capacity on a multiple-antenna channel," IEEE Trans. Commun., vol. 51, pp. 389-399, Mar. 2003.

[6] P. Nilsson and Z. Guo, "Algorithm and implementation of the K-best sphere decoding for MIMO detection," IEEE Journal on Selected Areas in Communications, vol. 24, pp. 491-503, Mar. 2006.

[7] B.M. Hochwald and S. ten Brink, "Achieving near-capacity on a multiple-antenna channel," IEEE Trans. Comms., vol. 51, pp. 389-399, Mar. 2003.

[8] S. Baro, J. Hagenauer, and M. Witzke, "Iterative detection of MIMO transmission using a list-sequential (LISS) detector," Proc. IEEE ICC '03, vol. 4, pp. 2653-2657, 11-15 May 2003.

[9] L. Tang, R. W. Liu, V. C. Soon, and Y. F. Huang, "Indeterminacy and identiability of blind identication," IEEE Trans. Circuits and Systems, vol. 38, pp. 499-509, May 1991.

[10] A. Medles and D. T. M. Slock, "Semiblind channel estimation for MIMO spatial multiplexing systems," in Proc. IEEE VTC2001-Fall, vol. 2, Oct. 2001, pp. 1240-1244.

[11] J. L. L. Morales and S. Roy, "On joint detection and channel estimation over rank-deficient MIMO links with sphere decoding," in 12th Canadian Workshop on Information Theory (CWIT), May 2011, pp. 165-169.

[12] W. Xu, Y. Wang, Z. Zhou, and J. Wang, "Joint ML channel estimation and data detection for STBC via novel sphere decoding algorithms," in Proc. IEEE VTC2005-Spring, May 2005, pp. 434-437.

[13] K. W. Wong, C. Y. Tsui, R. S. K. Cheng, and W. H. Mow, "A VLSI architecture of a K-best lattice decoding algorithm for MIMO channels," in Proc. IEEE International Symposium on Circuits and Systems (ISCAS), Aug. 2002, pp. III-273-III-276.

[14] Q. Li and Z. Wang, "Improved K-best sphere decoding algorithms for MIMO systems," in Proc. IEEE International Symposium on Circuits and Systems (ISCAS), May 2006, pp. 1159-1162. 